\title{
REVIEW
}

\section{Optical storage arrays: a perspective for future big data storage}

\author{
Min Gu, Xiangping $\mathrm{Li}^{*}$ and Yaoyu Cao*
}

The advance of nanophotonics has provided a variety of avenues for light-matter interaction at the nanometer scale through the enriched mechanisms for physical and chemical reactions induced by nanometer-confined optical probes in nanocomposite materials. These emerging nanophotonic devices and materials have enabled researchers to develop disruptive methods of tremendously increasing the storage capacity of current optical memory. In this paper, we present a review of the recent advancements in nanophotonics-enabled optical storage techniques. Particularly, we offer our perspective of using them as optical storage arrays for next-generation exabyte data centers.

Light: Science \& Applications (2014) 3, e177; doi:10.1038/lsa.2014.58; published online 23 May 2014

Keywords: big data centers; optical data storage; optical storage arrays; super-resolution

\section{INTRODUCTION}

Historically, the recording and storage of information have undergone a technological evolution from paintings to carvings, scribing and digitization, as illustrated in Figure 1. The major revolution in this developmental history is digitization, which allows music, sounds, pictures, words and movies to be stored in a binary form. The invention of optical compact discs (CDs), where a laser beam was adopted to store the binary data, was one of the major milestones in digital information technology. Together with magnetic storage devices, CDs have played a crucial role in the digital life of human beings.

Digital information technology has significantly boosted both economies and societies. Therefore, the storage of information from every sector, including education, entertainment, health, business and so on, has already become an indispensable part of the many activities of human beings in the information era. Consequently, humans are entering an era of information explosion. It has been estimated that the information generated by the major sectors is nearly doubled each year. According to a report prepared by International Data Corporation (IDC) in 2011, the total amount of data generated globally will reach $35 \mathrm{ZB}\left(1 \mathrm{ZB}=10^{3} \mathrm{~EB}=10^{6}\right.$ $\mathrm{PB}=10^{12} \mathrm{~GB}$ ) by $2020,{ }^{1}$ as shown in Figure 2. Like the information recorded in cave paintings, carvings and printings, the vast amount of digital information that has been stored constitutes a core portion of our civilization and history. However, there is a growing gap between the amount of digital data being created and the extent of available storage capacities. Big data centers with petabyte $(\mathrm{PB})$ and even exabyte (EB) capacities have emerged as the key enabling techniques and core platforms for cloud computing and storage, which have been heralded as the primary technical solution for the next generation of big data storage. ${ }^{2,3}$ However, the infrastructure of current information technology has been increasingly challenged by the limited storage capacity of current digital media as well as the shortcomings associated with the high energy consumption and short lifetime of such media.

As shown in the right-hand panel of Figure 2, current methods of data storage and archiving rely primarily on magnetization-based storage technologies, such as hard disk drives (HDDs) and magnetic tapes. To store vast amounts of information generated by clients, data centers composed of arrays of thousands of HDDs have been built. The sustainability of this technology is confronted by several crucial problems. First, the capacity of HDD units, which is less than $1 \mathrm{~TB}$ ( $1000 \mathrm{~GB})$ per disk, is far below the requirements of next-generation information storage. To increase the capacity to the PB scale, thousands of HDD units must typically be assembled, which means that the footprint of such a typical big data center is similar to that of a football stadium in size. Second, HDD-based data centers are not energy efficient. According to a statement issued by the US Environmental Protection Agency, data centers in the US alone consumed a significant amount of energy, accounting for $1.5 \%$ of the total US electricity consumption in 2010 at a cost of $\$ 4.5$ billion annually. Moreover, the amount of energy consumption is expected to nearly double within the next 5 years. Third, the limited lifetime of HDD technology requires data migration every 2 or 3 years to avoid any data loss. This significantly increases the energy-consumption burden, as a considerable amount of energy is consumed during such data migration. These challenges constitute fundamental obstacles to the longevity and sustainability of current information technology. Accordingly, technologies with capacities greater than PBs, ultralow energy consumption and ultralong lifetimes are in high demand to support big data centers.

Centre for Micro-Photonics, Faculty of Science, Engineering and Technology, Swinburne University of Technology, Hawthorn, VIC 3122, Australia

*These authors contributed equally to this paper

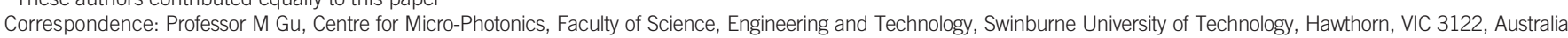
E-mail: mgu@swin.edu.au

Received 11 February 2014; revised 15 March 2014; accepted 23 March 2014 


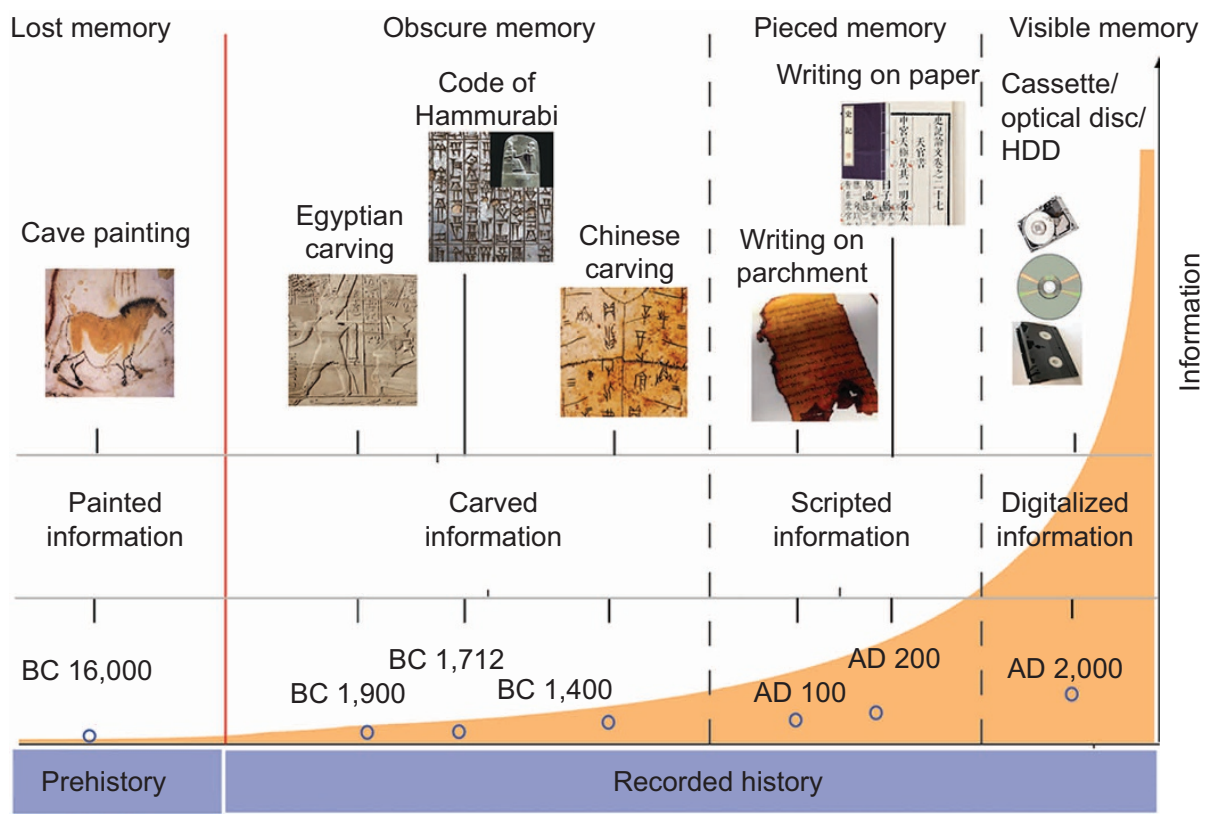

Figure 1 Historical evolution of the recording of information in human society. AD, Anno Domini; BC, Before Christ; HDD, hard disk drive.

On the other hand, optical data storage (ODS), which first emerged as CDs in the 1980s, is well known for its green features, including high storage capacities and high energy efficiencies compared with its counterparts. Unfortunately, current optical storage devices, including digital video discs (DVDs) and Blu-ray discs, can only record information in a diffraction-limited region within a layer beneath the surface of the disc. Therefore, their maximum capacity is limited to a few tens of gigabytes $(\mathrm{GBs}){ }^{4}$ As a matter of fact, optical discs have been sparsely used in current approaches to big data storage. Although tremendous research and development efforts have been expended on exploiting the volume of recording media for volumetric or multilayer memories facilitated by nonlinear excitation using a pulsed laser beam, ${ }^{5-7}$ the diffractive nature of light restricts the smallest recording bit to approximately half the wavelength of the light, and hence, the theoretical storage capacity in volumetric memories is limited to a few terabytes (TBs) per DVD-sized disc, ${ }^{8,9}$ which is still far below the requirements for massive data storage.

How can TB- or even PB-scale information be accommodated by a single DVD disc, and how can optical storage technology eventually overtake the storage capabilities of current HDDs? Revolutionary innovations in storage techniques are in high demand to transform conventional GB optical discs into ultrahigh-capacity storage media. In recent decades, nanophotonics has emerged as a rapidly expanding new field of light-matter interaction, largely owing to recent advances in nanotechnology that permit better control of material properties at the nanometer scale as well as the availability of sophisticated nanophotonic probes. As such, studies based on the new photonic principles have led to the development of artificial materials with negative refractive indices, ${ }^{10-12}$ nano-optical circuits, ${ }^{13,14}$ nanoscale light-emitting sources, ${ }^{15,16}$ imaging beyond the diffraction limit ${ }^{17-19}$ and superresolution optical lithography. ${ }^{20,21}$ These studies have laid the physical
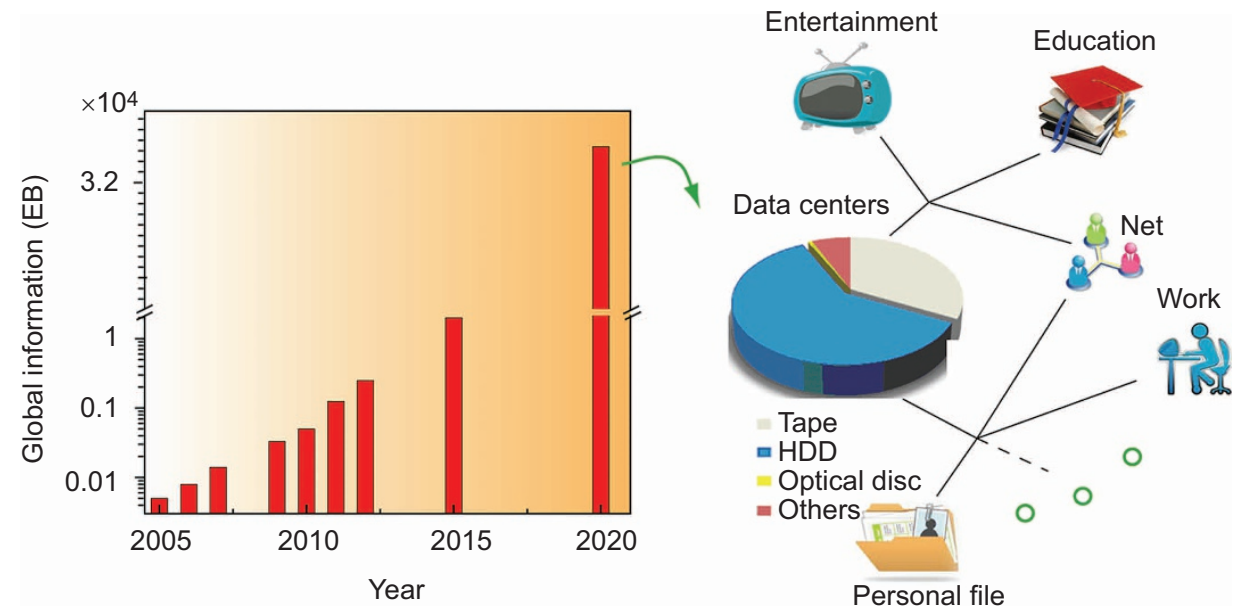

Figure 2 Global information generated each year. The right-hand panel shows the percentage of different storage methods used for storing large amounts of data (source data from IDC). HDD, hard disk drive; IDC, International Data Corporation. 


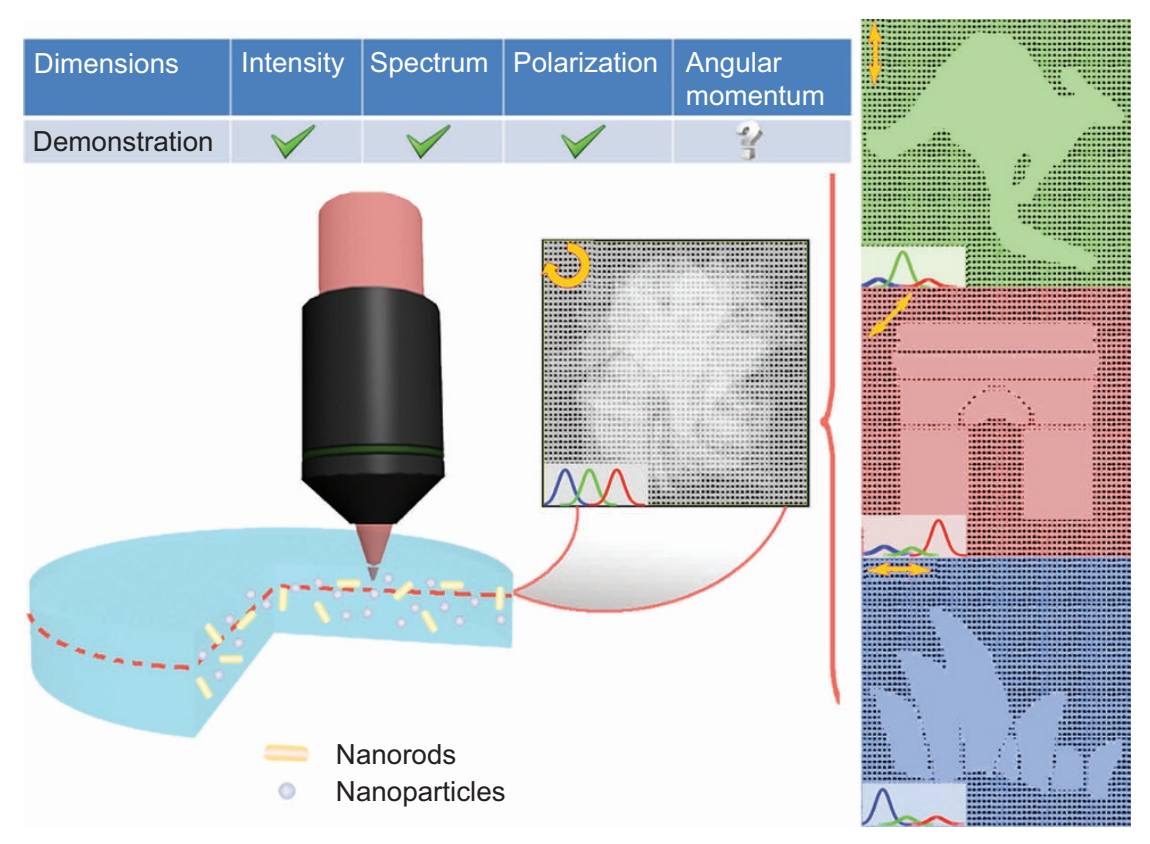

Figure 3 Scheme of multidimensional optical data storage. In the case of 5D data storage, the information bits are multiplexed in the polarization and spectrum domains of the writing beam and in multiple layers inside the medium. One recorded layer, indicated by the red dashed line and accessed using a randomly polarized broadband source as illustrated in the middle, cannot separate the polarization- and spectrum-coded information. The multiplexed information can be individually addressed by using the appropriate polarization state (indicated by the arrow) and wavelength, as illustrated in the right-hand column. ${ }^{4}$ The inset table lists four physical dimensions of the writing beam that could be employed for multiplexed storage. 5D, five-dimensional.

groundwork for the confinement of light-matter interactions to the nanometer scale, which paves the way toward breaking or circumventing the diffraction barrier and thus increasing storage capacity by using entirely new nanophotonic approaches.

\section{FEATURES OF NANOPHOTONICS-ENABLED OPTICAL DATA STORAGE}

Ultrahigh density

As has been known for some time, the limit on the optical resolution of a high-numerical-aperture (high-NA) objective, which was discovered by the German physicist and entrepreneur Ernst Abbe, sets a fundamental barrier that limits the smallest size of a recorded bit to approximately half the wavelength of the light used for recording in the lateral direction and approximately one wavelength in the axial direction. Hence, the theoretical maximum storage density for an aberration-free objective with a high NA of 1.4 is only on the order of TB per disc. ${ }^{8,9}$ Recent advances in nanophotonics can facilitate either the encoding of information in physical dimensions, such as those defined by the frequency and polarization parameters of the writing beam, or the achievement of three-dimensional (3D) superresolution recording, breaking the conventional storage-capacity limit.

\section{Multidimensional storage}

Nanophotonics allows for sharp color and polarization selectivity in light-matter interactions at the nanometer scale. For example, light can couple to surface plasmons, the collective oscillations of free electrons in metallic nanoparticles, which yield deterministic spectral responses associated with their sizes and shapes. ${ }^{22}$ These appealing properties make nanoparticles suitable for the implementation of spectrally encoded memory. Consequently, based on the principle of light-induced shape transitions, three-color spectral encoding by using gold nanorods of various sizes has been demonstrated. ${ }^{23}$ Similarly, polarization anisotropy can be created in nanocomposite materials by means of selective excitation by different polarization states of a writing beam. ${ }^{24,25}$ It has been recently demonstrated that when such polarization selectivity is combined with the sharp spectral selectivity of nanophotonics, gold nanorods can enable information recording in five dimensions, encoded across three wavelengths and two polarization states with an equivalent capacity of 1.6 TB in a single disc, ${ }^{23}$ as illustrated in Figure 3. Another possible dimension that can be exploited, which is also the most straightforward to control, is the intensity of the optical beam; control of the beam intensity forms the basis of the principle of gray-scale-encoded ODS. Instead of recording binary information, each voxel stores a range of gray-scale values by varying the discrete intensity levels of the writing beam. With a precise control of its focal intensities through vectorial Debye focusing of the beam using a high-NA objective, up to eight levels can be recorded and distinguished in photobleaching polymers ${ }^{26}$ and glass materials. ${ }^{27,28}$

The dimension of the beam that has received the least attention is the angular momentum (AM), which can be carried by photons in the forms of spin AM and orbital AM. As a demonstration of the principle of AM information multiplexing, the AM of light has been used to create orthogonal and spatially distinct streams of data-transmitting channels multiplexed in a single fiber. ${ }^{29}$ It is therefore expected that AM can be used to multiplex information and thereby boost the storage capacity. On the other hand, circularly polarized beams with different handedness (spin states) can be employed to introduce different magnetization orientations in all-optical magnetic recording. ${ }^{30}$ In this case, if the recording spots could be reduced to their smallest, diffraction-limited size, ${ }^{31}$ the optical magnetic response could act as another dimension to allow for further increase in the storage capacity. Limited by the material response, the multiplexing of information in six individual dimensions has never been achieved. Recent advances in nanotechnology, which have enabled a variety of new nanocomposites, such as nitrogen vacancy centers in diamonds ${ }^{19,32,33}$ with optically detected magnetic resonance, ${ }^{34,35}$ might provide a solution for 


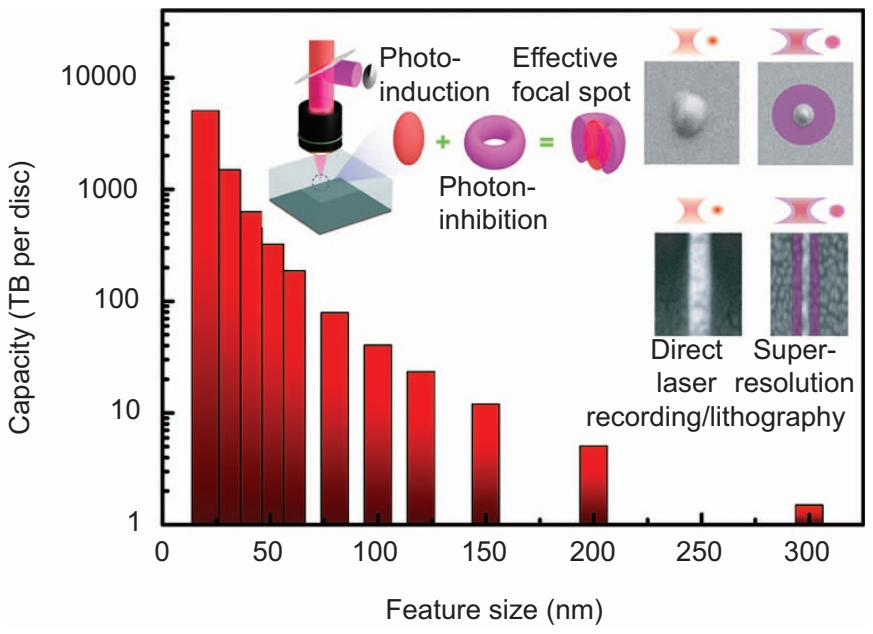

Figure 4 Projection of the maximal capacity that a single disc can hold as a function of the feature size of the recorded bits. In the projection, the lateral separation and the axial separation are set to 2.5 times and 8 times the feature size, respectively. The insets illustrate the principle underlying SPIN's ability to break the diffraction barrier and achieve super-resolution recording/lithography. Top inset: comparison of direct laser recording and super-resolution recording by using SPIN methods. Bottom inset: comparison of conventional laser lithography and super-resolution lithography. SPIN, super-resolution photoinduction-inhibition nanolithography.

exploiting the maximal number of information channels by simultaneously employing all physical dimensions of the writing beam.

\section{$3 D$ super-resolution recording}

A variety of methods have been proposed and demonstrated to break the diffraction barrier in the near-field region and achieve superresolved areal memories. ${ }^{36,37}$ However, these approaches do not exhibit the ability to record information in the volume of a medium. Recently, inspired by a diffraction-unlimited far-field imaging approach, ${ }^{38}$ scientists have developed super-resolution photoinduction-inhibition nanolithography (SPIN), which can break the diffraction barrier and achieve 3D super-resolved writing.

In contrast to conventional optical memory, SPIN is achieved by employing dual beams during recording; the behavior of each beam is still governed by its diffraction. In general, the two beams operate at different frequencies enduing different functions, as illustrated in the insets of Figure 4. One of the two beams, with a Gaussian shape, falls within the transition bands of the materials and thus is responsible for photoinduction. The other beam, with a spatially modulated intensity distribution that is usually a doughnut shape (with zero light intensity in the center), is responsible for inhibiting photoinduction everywhere in the focal region except at its center. Consequently, the effective focal spot can be made much smaller than the diffraction barrier by the spatial superposition of the two beams and varying the intensity ratio between the two beams.

By employing the principle of SPIN, researchers have successfully demonstrated super-resolved line fabrication as well as dot recording in a variety of photoinduction systems, as schematically illustrated in the right-hand panel of insets in Figure 4. Table 1 summarizes the state of the art of SPIN-based super-resolution recording methods for the production of ultrafine features. In general, photoinduction can refer to any photoinduced chemical or physical processes that initiate a change in material properties, such as photochromism, ${ }^{39}$ photopolymerization ${ }^{20}$ and photoreduction, ${ }^{48}$ and that can also be terminated by an inhibition beam operating at a different wavelength. As such, the photochromism process has been used to inhibit photoabsorption, and the smallest line-feature size of $30 \mathrm{~nm}$ (approximately one-tenth of the wavelength $\lambda$ ) has been successfully demonstrated ${ }^{39}$ Recently, a photopolymerizable material with improved photosensitivity and mechanical stability has been developed and successfully applied in using the SPIN method for the fabrication of suspended lines with the smallest feature size down to $9 \mathrm{~nm}$, or $\lambda / 42 .{ }^{20}$ These exceptional features open a new avenue toward diffraction-unlimited laser fabrication, as well as for the development of ultrahigh-density optical memory.

The development of 3D super-resolved writing methods will enable further decrease in the effective size of recorded bits to below $50 \mathrm{~nm}$ and will eventually boost disc capacity to approach or even surpass the $\mathrm{PB}$ scale in the near future, as projected in Figure 5. In the meantime, sophisticated techniques for light manipulation on the focal plane are necessary to maintain an effective focal spot smaller than the diffraction limit with enriched physical dimensions. The combination of super-resolution techniques and multiplexing in the physical dimensions can further expand disc storage capacity beyond hundreds of PBs. Clearly, nanophotonics-enabled storage techniques are far superior to the current HDD and flash memory techniques, which are encountering density bottlenecks (Figure 5).

\section{Ultrahigh throughputs}

Another challenge confronting big data storage is low writing and reading throughputs. Based on the current throughput $\left(\sim 20 \mathrm{MB} \mathrm{s}^{-1}\right)$ of Blu-ray discs, it might take more than one and a half years to access $1 \mathrm{~PB}$ of information. ${ }^{3}$ Even taking the current HDD technique into consideration $\left(\sim 100 \mathrm{MB} \mathrm{s}^{-1}\right)$, it would still require more than 100 days to access such a massive amount of data. Therefore, it is equally as

Table 1 The development of SPIN methods

\begin{tabular}{|c|c|c|c|c|}
\hline Photoinhibition methods & Wavelength of photoinduction (nm) & Wavelength of photoinhibition ( $\mathrm{nm}$ ) & Lateral feature size (nm) & Pattern \\
\hline Photodeactivation ${ }^{21}$ & 800 & 800 & $\sim 100(1 / 8 \lambda)$ & Dots \\
\hline Photochromism ${ }^{39}$ & 325 & 633 & $30(1 / 10 \lambda)$ & Lines \\
\hline Photoradical $^{40}$ & 473 & 364 & $64(1 / 8 \lambda)$ & Dots \\
\hline Photoluminescence ${ }^{41}$ & 800 & 532 & $65(1 / 12 \lambda)$ & Lines \\
\hline Photoradical $^{42}$ & 488 & 375 & $40(1 / 12 \lambda)$ & Dots \\
\hline Photodeactivation $^{43}$ & 800 & 800 & $\sim 150(1 / 6 \lambda)$ & Lines \\
\hline Photoluminescence ${ }^{44}$ & 800 & 532 & $\sim 120(1 / 7 \lambda)$ & Lines \\
\hline Photoabsorption $^{45}$ & 800 & 642 & $\sim 120(1 / 7 \lambda)$ & Dots \\
\hline Stimulated emission depletion ${ }^{46}$ & 780 & 532 & $55(1 / 14 \lambda)$ & Lines \\
\hline Photoradical $^{20}$ & 800 & 375 & $9(1 / 42 \lambda)$ & Lines \\
\hline Photoswitching 47 & 405 & 491 & 200 (resolution) & Dots \\
\hline
\end{tabular}

*SPIN, super-resolution photoinduction-inhibition nanolithography. 


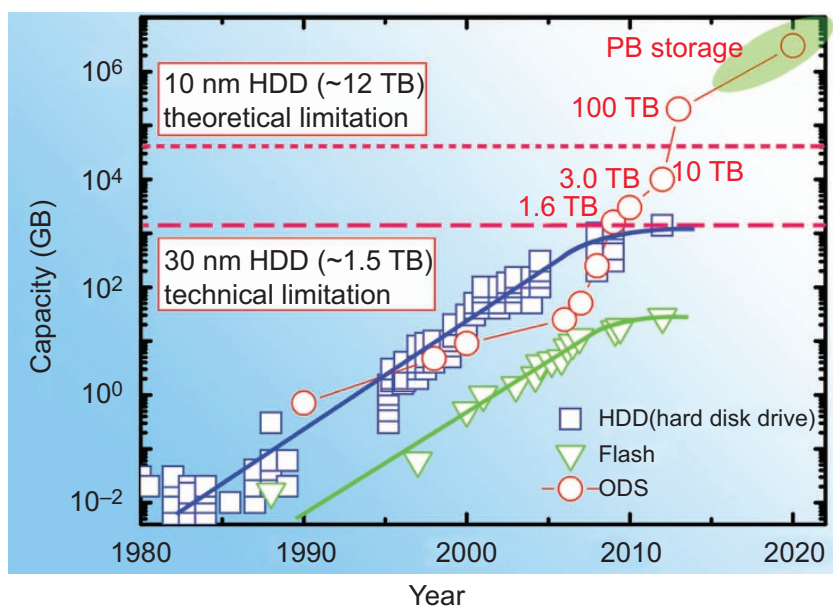

Figure 5 Comparison of the development of storage capacities using the HDD (squares), flash (triangles) and ODS (circles) techniques. The capacity of flash memory is calculated based on a storage area of $1 \mathrm{~cm}^{2}$. The HDD technique is currently subject to a $30 \mathrm{~nm}$ technical limitation ( 1.5 TB) and is expected to approach its theoretical limitation of $10 \mathrm{~nm}(\sim 12 \mathrm{~TB})$. However, 5D storage has already broken the technical limitation of the HDD technique, achieving a capacity of 1.6 TB in 2009. ${ }^{23}$ The super-resolution focal-volume technique has pushed the capacity further to 3 TB. ${ }^{9}$ Moreover, the application of the SPIN method in 3D optical storage has enabled a feature size below $100 \mathrm{~nm}$ with an equivalent capacity of approximately $10 \mathrm{~TB} .{ }^{49}$ The latest result of a recorded bit size of $32 \mathrm{~nm}$ has surpassed the theoretical limitation of the HDD technique and demonstrated a new world record with an equivalent capacity of approximately 100 TB. ${ }^{50}$ 3D, three-dimensional; 5D, five-dimensional; HDD, hard disk drive; ODS, optical data storage; PB, petabyte; SPIN, super-resolution photoinductioninhibition nanolithography.

important to boost writing/reading throughputs as it is to increase storage capacity.

Various optical parallelism methods for generating multifocal arrays in single laser shots have been proposed and experimentally demonstrated, including microlens arrays, ${ }^{51}$ diffractive optical elements, ${ }^{52}$ Debye-based Fourier transformation ${ }^{53}$ and dynamic computer-generated holograms. ${ }^{54}$ Among these methods, Debye-based Fourier transformation enables the formation of diffraction-limited multifocal spots using a high-NA objective wherein each focal spot can be dynamically programmable, which is a necessity for ultrahigh-density optical recording. The implementation of this method by using a spatial light modulator for parallel recording is illustrated in Figure $6 a^{53}$ The single beam carrying the modulated phase pattern calculated by the Debye-based Fourier transform can be diffracted into the designed multifocal array in the focal plane of the objective. With a few hundred programmable focal spots for parallel writing, the throughput of optical storage could be significantly increased to $\sim 10 \mathrm{~GB} \mathrm{~s}{ }^{-1}$; therefore, the accessing time for $1 \mathrm{~PB}$ could be dramatically reduced to only a few days. The Debye-based Fourier transform method can generate not only in-plane multifocal arrays but also highquality $3 \mathrm{D}$ multifocal arrays for volumetric parallel writing, ${ }^{55}$ and it can even be integrated with other physical parameters to increase the throughput beyond tens of GB s ${ }^{-1}$, as illustrated in Figure 6b. Thus, polarization states of individual focal spots in $3 \mathrm{D}$ multifocal arrays have been controlled to achieve parallelism in the dimension of the polarization states. As an example, the generation of cylindrically polarized multi-focal arrays has been demonstrated by applying the vectorial Debye-based Fourier transform method. ${ }^{56}$ Furthermore, the combination of polarization-controllable multifocal arrays with SPIN methods can allow for super-resolution parallel recording and readout, which will therefore be a core platform for the development of PB optical discs (Figure 6c).

\section{Ultrahigh security}

The physical dimensions of a writing beam can be used not only as information channels to increase the storage capacity but also as versatile means to encrypt information for data security, which is one of the most important aspects of any memory system.

As an electromagnetic wave, light can selectively interact with optical materials with physical anisotropic properties via its polarization, or the oscillation of its electric fields. The response of optical materials strongly depends on the orientation of the electric dipoles with respect to the polarization state of the light. As such, information can be encrypted using a specific polarization state of a writing beam, which cannot be retrieved without pre-knowledge of the polarization key. By rotating the polarization orientation, flexibility of the encryption key can be achieved, and multiple states of information can be encrypted in the same spatial region. Thus, information can be

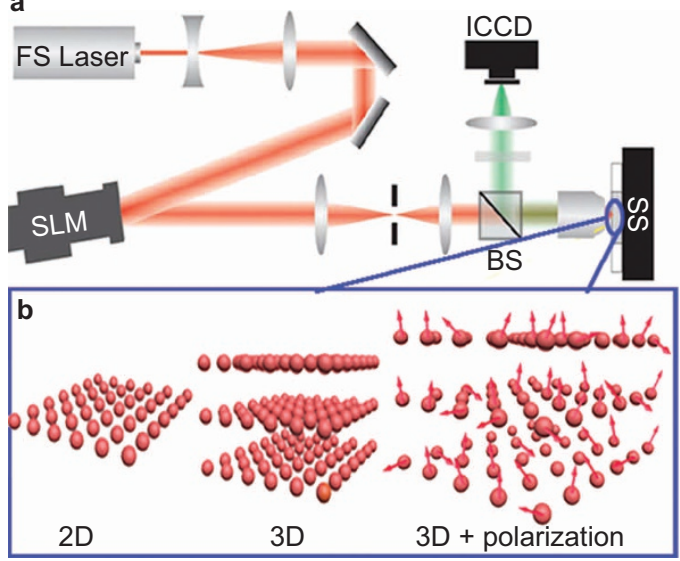

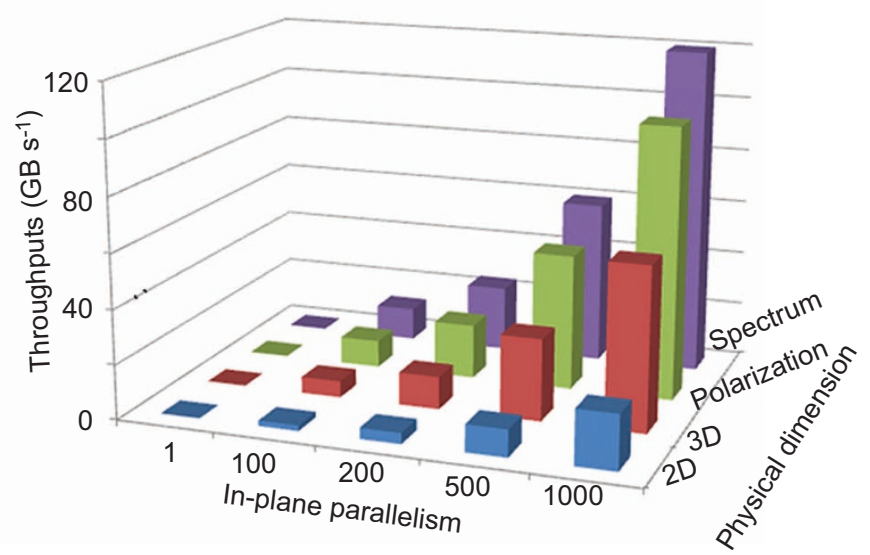

Figure 6 (a) Scheme for parallel optical recording using an SLM. (b) Schematic illustrations of in-plane parallel (2D) recording, volumetric 3D recording and 3Dcombined-with-polarization parallel recording. The arrows indicate the polarization orientations. (c) The overall throughput as a function of the in-plane parallelism and physical dimensions. BS, beam splitter; 2D, two-dimensional; 3D, three-dimensional; FS, femtosecond; ICCD, intensified charge-coupled device; SLM, spatial light modulator; SS, scanning stage. 


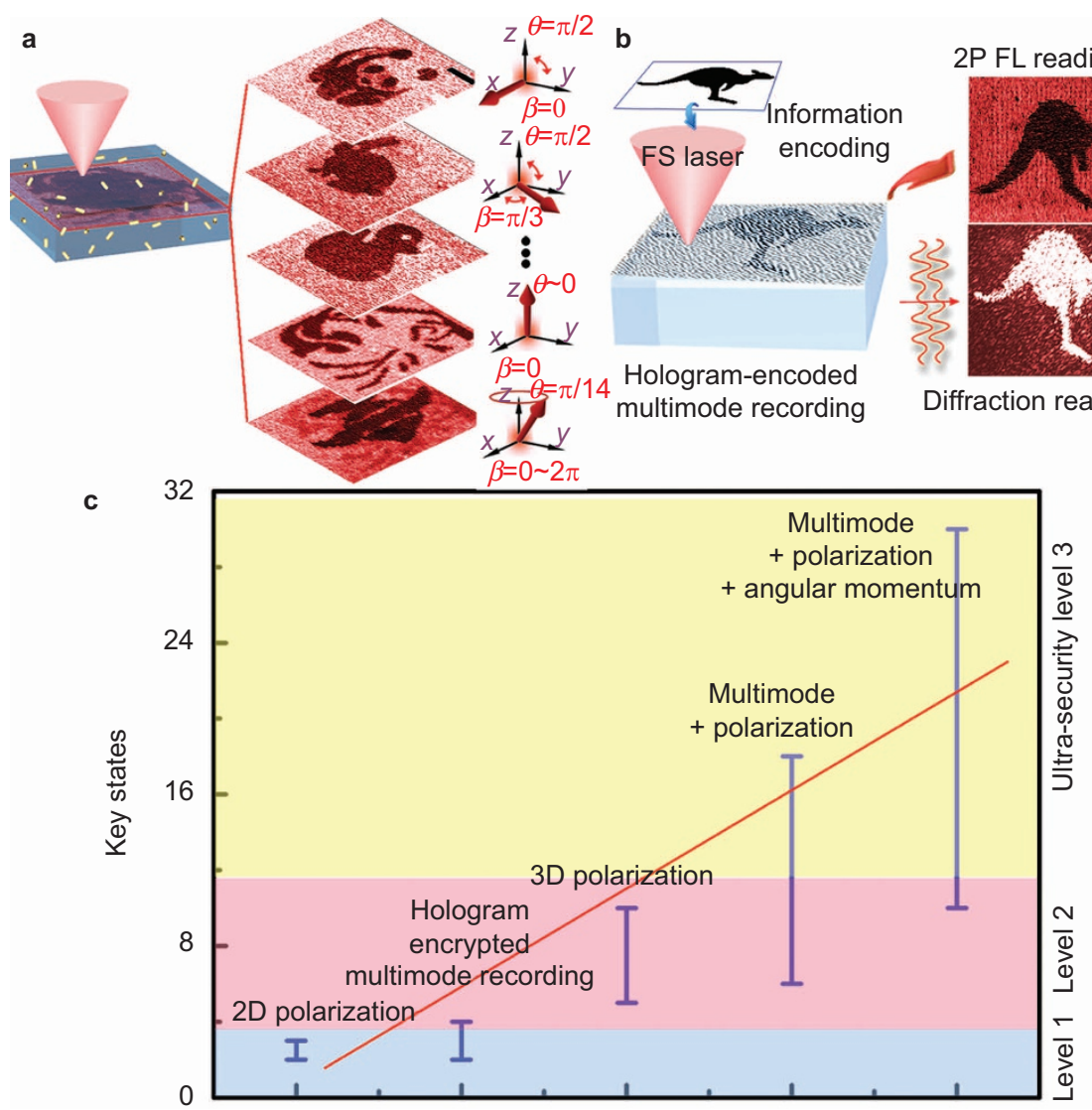

Figure 7 (a) Scheme for 3D polarization encryption using 3D oriented polarization states. ${ }^{60}$ (b) Illustration of hologram-encoded multimode recording for enhanced data security. Information bits can be randomly accessed through the $2 \mathrm{P}$ fluorescence readout and retrieved in the diffraction mode of the recorded holograms. ${ }^{61}$ (c) Projection of levels of information security by combining multimode recording with other physical dimensions. 3D, three-dimensional; FL, fluorescence; FS, femtosecond; 2P, two-photon.

encrypted in the two polarization states of writing beams at $0^{\circ}$ - and $45^{\circ}$ oriented polarizations through polarization-induced birefringence. ${ }^{24}$ Recently, it has been demonstrated that by employing the depolarization effect through the tight focusing of a radially polarized beam, one can generate a longitudinal polarization state within the focal region. ${ }^{57,58}$ Through the superposition of weighted radially and azimuthally polarized beams, a 3D arbitrary polarization orientation can be achieved in the focal region of a high-NA objective. In combination with the sharp polarization sensitivity of gold nanorods, 3D polarization encryption within a solid angle in the focal plane has been demonstrated for ultrahigh security, ${ }^{59}$ as illustrated in Figure 7a.

Additionally, the enrichment of the physical dimensions for lightmatter interactions offered by nanophotonic approaches enables information to be recorded in multimode material responses, which opens a new avenue toward a high level of information security. For example, simultaneous changes in the refractive index and fluorescence intensity of graphene-based materials have been demonstrated to hold the potential for hologram-encoded multimode recording, ${ }^{61}$ as shown in Figure $7 \mathrm{~b}$. On the one hand, the information can be randomly accessed through two-photon fluorescence readout. On the other hand, the information can be simultaneously encrypted in holograms, ensuring its integrity and durability. It is possible to integrate hologram-encrypted multimode recording with other physical dimensions, such as polarization and AM, to achieve an even higher level of information security, as illustrated in Figure 7c. However, the experimental achievement of such a high level of security strongly depends on material properties of the recording medium, specifically, its deterministic responses to light with various characteristics. Nanotechnology that offers the ability to engineer and control material properties on the nanoscale may provide a solid platform for future ultrahigh-security optical storage.

\section{Ultralong lifetime}

As we have noted, magnetization-based HDD techniques have a limited lifetime of $2-5$ years, and therefore, frequent data migration is needed to avoid potential data loss. However, ODS has a longer lifetime than HDDs, which dramatically reduces the required frequency of data migration. In addition, ODS only consumes energy when the data are written or read out and does not consume any energy when the optical disc is in idle state. For comparison, Figure 8 illustrates the power consumption and operational cost of information storage incurred by a single HDD (4480 kWh) and a single disc ( $35 \mathrm{kWh})$ over 50 years. Clearly, optical technology greatly reduces the waste generated by frequent data migration, reduces the energy consumption in idle status and reduces the cost for the replacement of new units associated with short lifetimes. Moreover, increasing the lifetime of ODS to greater than 50 years can dramatically increase the savings in overall expenditures, including electricity and costs for storage devices, by more than $96 \%$ compared with HDD techniques.

Consequently, the development of ODS with ultralong lifetimes has been a subject of intensive research. Permanent laser-induced physical changes, such as voids in polymers ${ }^{62}$ and glass materials, ${ }^{63}$ provide an 


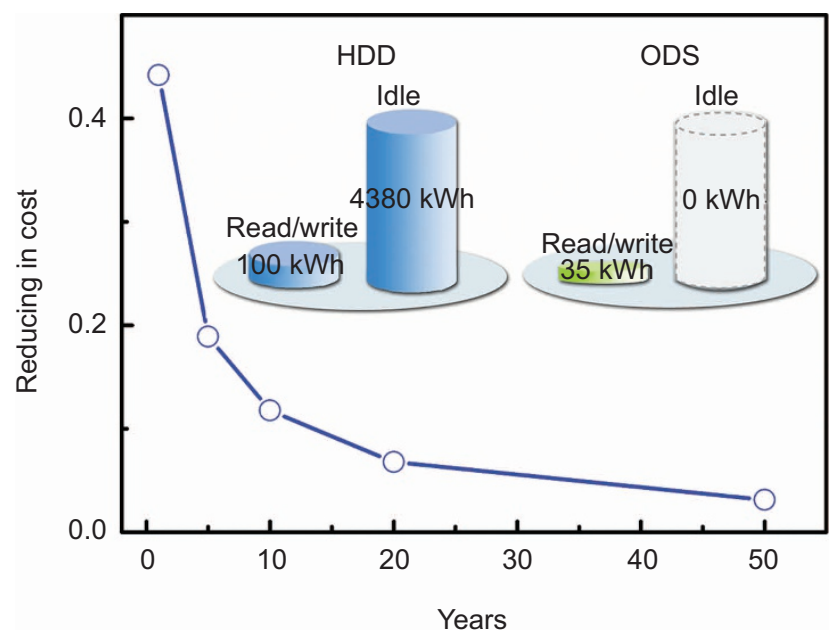

Figure 8 Reduction in the operational cost for a single ODS unit compared with that of a single HDD unit as a function of years elapsed. The inset shows a comparison in terms of the electricity consumption for reading/writing and in idle status for a single unit over 50 years. In this comparison, a single HDD unit typically consumes $20 \mathrm{~W}$ for reading/writing, and it is used in this manner for $100 \mathrm{~h}$ per year. In the idle status, it consumes $10 \mathrm{~W}$ per hour. A single ODS unit consumes $7 \mathrm{~W}$ for reading/writing and is used in this manner for $100 \mathrm{~h}$ per year. The cost is calculated based on an assumption of $\$ 0.12$ per $\mathrm{kWh}$, and the unit prices of the HDD and the ODS are $\$ 80$ and $\$ 40$, respectively. HDD, hard disk drive; ODS, optical data storage.

approach to long-lifetime storage without information degradation. Laser inscriptions in glass materials ${ }^{27,28}$ can withstand temperatures of up to $1000{ }^{\circ} \mathrm{C}$ and maintain data stability and readability for up to thousands of years, in principle. The demonstrated physical changes including voids and inscription are limited by a low capacity of approximately $50 \mathrm{~GB}$ per disc because the physical size of each bit cannot be smaller than the diffraction barrier. The top-down nanocomposite approach offers an alternative method that may allow the development of SPIN methods to break the diffraction limit for ultrahigh capacity ODS with thousands of years of lifetime.

\section{OPTICAL STORAGE ARRAYS: A PERSPECTIVE FOR EB DATA CENTERS}

The question of how to store the vast amount of data generated each year completely and efficiently is an urgent challenge confronting current information technologies. For example, the Australian telescope generates more than $700 \mathrm{~TB}$ of data every day. Scientists must selectively store only a portion of these valuable data. The US national priority project to map the human brain to gain an understanding of the cell types present in the brain, how they are connected and how they communicate is expected to generate more than $1000 \mathrm{ZB}$ of data. It is impossible to store such a vast amount of information using current technologies, even the cloud computing and storage have emerged as technical solutions for future big data storage, in which PB data centers provide key enabling platforms. Since the capacity of a single HDD is no larger than $1 \mathrm{~TB}, \mathrm{~PB}$ data centers must typically be built on arrays of thousands of HDDs. Because of the low capacity and high energy consumption of HDD techniques, the resources necessary to store large amounts of data require inordinate amounts of power and rack space. A typical PB data center occupies a stadium-sized space, including the racks and cooling accessories, which represents a significant overhead for such a data center. According to the IDC report, ${ }^{1}$ the annual electricity consumption and maintenance costs for such a PB data center based on HDD arrays could even surpass the cost of the hardware itself.

Recent advances in nanophotonics-based ODS have exhibited the ability to address the bottlenecks that confront current HDD techniques, including storage capacity, lifetime, energy consumption and data-transfer rates. In particular, the SPIN method has enabled ODS with a capacity approaching PBs per disc. Each such disc may potentially replace a stadium-sized $\mathrm{PB}$ data center in terms of capacity, as schematically illustrated in Figure 9. The integration of a single nanophotonics-based ODS unit with PB-scale capacity into multiple-unit arrays will provide a new platform for EB-scale big data centers to cope with vast amounts of information being produced in the digital era. Combined with its green features in energy conservation, nanophotonics-enabled optical storage arrays (OSAs) hold the potential to switch from the current magnetization-based approach to big data storage to the use of optical discs.

Most importantly, photonics has been heralded as a sustainable information technique to replace its current electronic- and magnetic-based counterparts for big data era. It is believed that optical chips will emerge in the following 5-10 years to replace electronic chips for ultrafast and ultralow-energy-consumption optical computing. The optical fiber has already replaced copper-based wires for ultrahigh speed and ultrahigh-energy-efficiency communication. The emergence of OSAs for ultrahigh-capacity information storage will complete the optical information-technology chain to create an entirely new approach to all-optical cloud computing/storage toward a sustainable future. Once that day comes, the efficient and complete storage of big data will affect every aspect of a normal person's life, including e-health, e-banking, e-education and e-defense. It will completely change the way people consume and store information.

The core of the OSA system is based on nanophotonics-enabled PB ODS units. The system consists of $\mathrm{N}$ units of $\mathrm{PB}$ drives and discs controlled by a server through a buffer, as schematically illustrated in Figure 9a. The capacity of the entire array system is the product of the total number of PB units and the capacity of each single disc. For example, the storage capacity of such an array system can be significantly increased to the EB or even zettabyte (ZB) scale by increasing the number of PB ODS units present in the array, as shown in

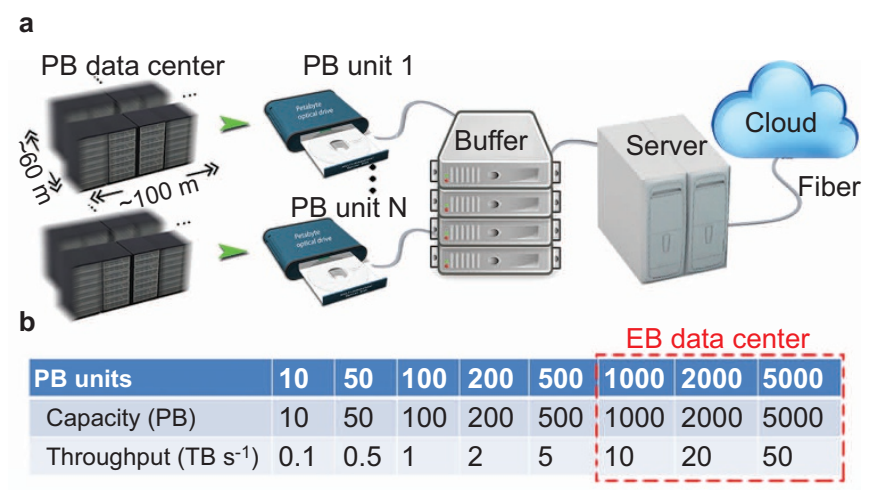

Figure 9 (a) Schematic illustration of an OSA based on integrating $N$ units of nanophotonics-enabled PB ODS devices, where each single PB ODS unit is capable of replacing a conventional PB-scale big data center based on HDD arrays of $\sim 100 \mathrm{~m}$ by $60 \mathrm{~m}$ in size. (b) The capacity and throughput of the storage array system as a function of the number of ODS units. Big data centers with a capacity approaching EBs become possible using the new OSA technique without any increase in the infrastructure size of the data centers. EB, exabyte; HDD, hard disk drive; ODS, optical data storage; OSA, optical storage array; PB, petabyte. 


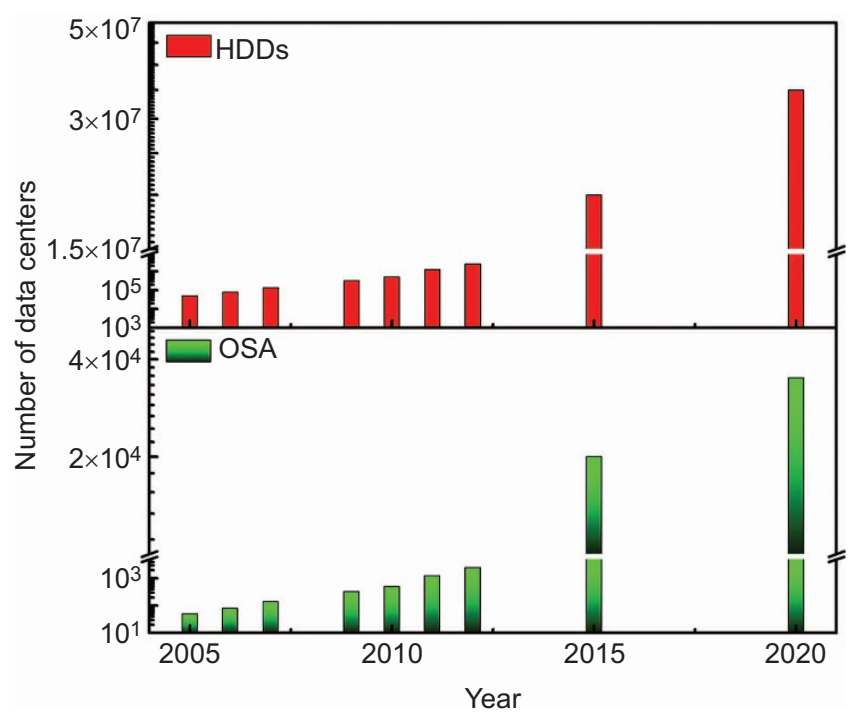

Figure 10 Number of data centers based on 1000 HDD units (red bars) and 1000 OSA units (green bars) required to store the global information generated each year. HDD, hard disk drive; OSA, optical storage array.

Figure $9 \mathrm{~b}$. In the meantime, each unit has the ability to write and read in optical parallelism to maximize the throughput. The overall throughput of the array system is a product of the total number of units and the optical parallel throughput of each single drive, which can far exceed TB s $s^{-1}$, as shown in Figure 9b.

\section{Ultrahigh capacity and ultrahigh throughput of big data centers enabled by OSA}

According to the IDC's projections of the global information generated each year in Figure 2, millions of PB data centers are required to store such vast amounts of information based on the current HDD technique. By switching the future big data centers to the approach of nanophotonics-enabled OSA, each single disc will be able to achieve $\mathrm{PB}$ capacity and will therefore replace one PB-scale data center based on HDD arrays. With a similar number of discs, an OSA can reach far beyond PB-scale and even EB-scale capacity. For comparison, an HDD array consisting of 1000 units has a maximum capacity of $1 \mathrm{~PB}$, whereas a nanophotonics-enabled OSA with the same number of units can exceed $1 \mathrm{~EB}$, and even approaching the $\mathrm{ZB}$ scale is possible. It can be seen that OSAs can significantly reduce the number of big data centers required to satisfy the capacity requirement by more than three orders of magnitude, as shown in Figure 10.

In addition to their exceptionally high capacity, future big data centers based on OSAs enabled by nanophotonic parallel recording and reading techniques will permit ultrahigh throughput beyond the TB s ${ }^{-1}$ level. As shown in Figure 6, if the multifocal array technique were integrated with the technique of multiplexing in physical parameters, the throughput of each single PB unit disc could exceed $10 \mathrm{~GB} \mathrm{~s}^{-1}$. A typical OSA with 1000 units could easily achieve a throughput of greater than $10 \mathrm{~TB} \mathrm{~s}^{-1}$, which is far superior to the current HDD array technique with its typical throughput of a few tens of $\mathrm{GB} \mathrm{s}^{-1}$. Unlike its counterparts, OSA-based big data centers are fully compatible with the optical-fiber communication technique, which is ubiquitously used in big data centers. By removing the necessity of electrical-to-optical converters, the data transfer between remote terminations and storage media could be made even faster.

\section{Ultrahigh energy efficiency}

In addition to the question of how to accommodate an explosively increasing amount of information in current storage devices, another major challenge confronting the current big data centers based on HDD arrays is the question of how to store information in an energy-efficient manner. Because of the low areal density of present technology, current data centers must be built on arrays of thousands of HDDs in order to reach a capacity of PBs. Moreover, HDD arrays consume energy during both operating and idle states and produce a significant amount of heat, which must be dissipated by gigantic accessorial cooling systems that consume a tremendous amount of electricity. On the one hand, this means that a single HDD-based data center occupies a footprint comparable to that of a stadium ( $100 \mathrm{~m}$ by $60 \mathrm{~m})$. On the other hand, the high energy consumption raises a number of serious concerns regarding the sustainability of big data centers, including the vast costs of the infrastructure and environment pollution as well as the ever-increasing cost of maintenance. By contrast, nanophotonics-enabled OSAs of ultrahigh capacity, approaching the PB scale in a single DVD-sized disc, can dramatically ameliorate these concerns.

The ultrahigh capacity and compactness of OSAs can dramatically alleviate the costs for the infrastructures of such stadium-sized big data centers. It is reasonable to project that a PB-scale optical storage unit based on nanophotonics-enabled recording methods will be developed in dimensions of $200 \mathrm{~mm} \times 125 \mathrm{~mm} \times 36 \mathrm{~mm}$ within the following 5-10 years. Most importantly, OSAs do not consume energy, while they are in the idle state, which eliminates the necessity for cooling accessories. For the same capacity of $1000 \mathrm{~PB}$, the use of OSAs can reduce the data-center size compared with that required for a data center based on HDD arrays by three orders of magnitude. As shown in Figure 11a, OSA technology represents a tremendous reduction in the infrastructure required for big data centers to satisfy the demands of storing vast amounts of information newly generated each year. Taking as an example an electricity consumption of $1.0 \mathrm{kWh}$ per effective TB for current HDD-based data centers (0.8-1.8 $\mathrm{kWh} \mathrm{TB}^{-1}$, including storage and cooling costs) and assuming that the infrastructural development of a new $\mathrm{PB}$ data center will consume $\sim 50 \%$ of the yearly operating cost, Figure $11 \mathrm{~b}$ and $11 \mathrm{c}$ represents the reduction of the infrastructural development of new $\mathrm{PB}$ data centers, in terms of equivalent electricity consumption as well as $\mathrm{CO}_{2}$ emission by switching to the OSA technique to satisfy the requirement of the global data consumption. Indeed, the OSA enabled by nanophotonic approaches can save trillions of kWh of electricity per annum on the infrastructure development of new data centers, as well as eliminate millions of tons of $\mathrm{CO}_{2}$ emission for a greener future.

In addition, OSAs can significantly reduce the annual electricity consumption required to store vast amounts of information generated each year. Per effective TB of data, current HDD-based data centers consume $\sim 1.0 \mathrm{kWh}$ of electricity for data storage. Based on IDC projections, more than 300 trillion $\mathrm{kWh}$ of electricity will be consumed to store the newly generated data in the year 2020 if HDD technology is used, as shown in Figure 11d. For comparison, the OSA technique, with its ultralow energy consumption per writing cycle and zero consumption, while in the idle state, can significantly reduce the electricity consumption and increase the efficiency of energy usage. The electricity conserved in the year 2020 by switching to OSA techniques could be equivalent to one thousand times the US residential electricity consumption in 2011.

\section{Ultralong-lifetime archiving}

The permanent archiving of information that is generated annually is another important aspect of big data storage. Typically, data stored 

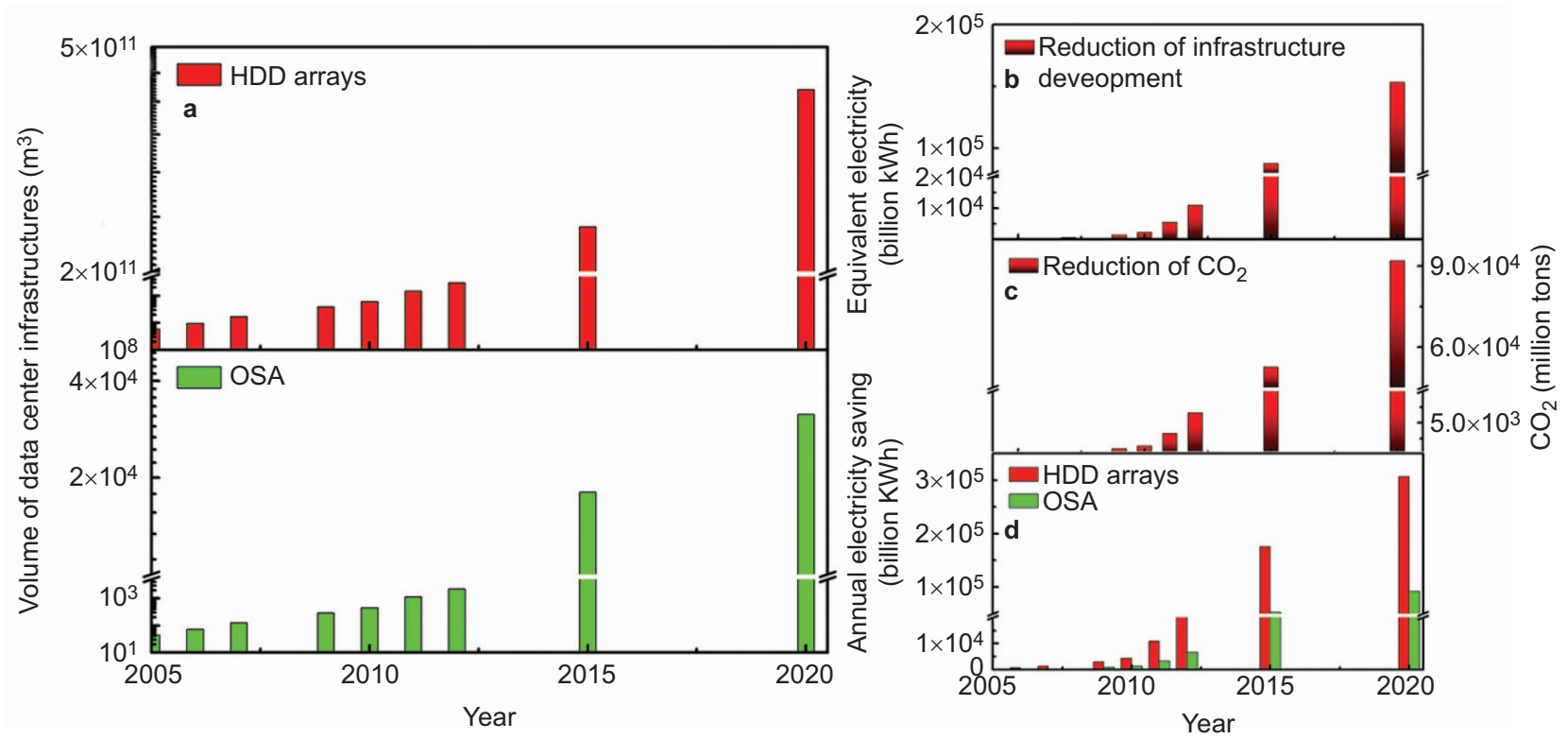

Figure 11 (a) Comparison of the infrastructure volumes of data centers based on HDD arrays and OSAs, which are required to store the information generated each year according to the IDC projection. The annual reduction offered by the infrastructural development of new PB data centers is expressed in terms of (b) equivalent electricity consumption and (c) $\mathrm{CO}_{2}$ emissions ( $1 \mathrm{kWh}$ electric power $=0.6 \mathrm{~kg} \mathrm{CO}_{2}$ emission ${ }^{64}$ ). (d) Annual electricity consumption of data centers based on $\mathrm{HDD}$ arrays (red squares) and OSAs (green squares) for the storage of the information generated each year, according to IDC projections. HDD, hard disk drive; IDC, International Data Corporation; OSA, optical storage array; PB, petabyte.

using HDD-array techniques must be migrated every 2 or 3 years to prevent data loss. This requirement raises the concern of large amounts of energy consumed during such frequent data migration; as the capacity of data centers expands, this energy consumption becomes an increasingly significant obstacle to the longevity of current big data centers. Since nanophotonics-enabled storage methods allow write-once-read-many protocols, OSAs can alleviate the ongoing electricity demand of big data centers for long-lifetime data persistence. In addition, the data stored in optical discs does not consume any energy unless the information is written or read out during their lifetimes, which also tremendously reduces the operational and maintenance costs. For comparison, the storage of one effective TB of information in $\mathrm{PB}$ optical discs using nanophotonic approaches consumes less than $0.3 \mathrm{kWh},{ }^{4}$ which represents an energy savings of more than $70 \%$ in a single writing cycle. Optical storage discs with 100-year lifetimes can reduce the energy consumed for storage by more than $99.4 \%$ compared with HDD arrays, which require 50 data transfers in a 100-year information archive, as shown in Figure 12. This feature could result in astronomical figure of energy conservation if all data generated in the year 2020 could be stored using OSA techniques and archived for more than a century, and the energy conservation offered by OSAs with lifetimes of thousands of years or more could be even more pronounced.

\section{OUTLOOK}

In this paper, we have summarized the recent developments in nanophotonics-enabled optical storage techniques. Nanophotonic approaches enable light-matter interaction at the nanoscale and the enrichment of the physical dimensions used for encoding, thereby providing an avenue toward ultrahigh-density $\mathrm{PB}$ optical storage with ultrahigh security and ultrahigh throughput. The integration of single nanophotonicsenabled storage units offers new prospects for all-optical big data centers to address the bottlenecks in capacity and energy efficiency experienced by current big data centers based on HDD arrays. Because of their exceptional features, OSAs exhibit tremendous potential for big data storage and long-lifetime archiving.

Moreover, a data center based on OSAs is capable of maintaining higher standards of integrity and functionality for its stored information than its counterparts based on HDD arrays. Unlike electronic- or magnetic-based storage techniques, OSAs are robust to a variety of harsh environmental conditions (temperatures of $10-40{ }^{\circ} \mathrm{C}$, humidity of $20 \%-80 \%$ ) and resistant to data corruption caused by radiation, making them ideal for many space and military applications. In particular, one potential application of such data centers is the storage of confidential information such as that related to banks, governments and national defense. To avoid malicious tampering with data integrity

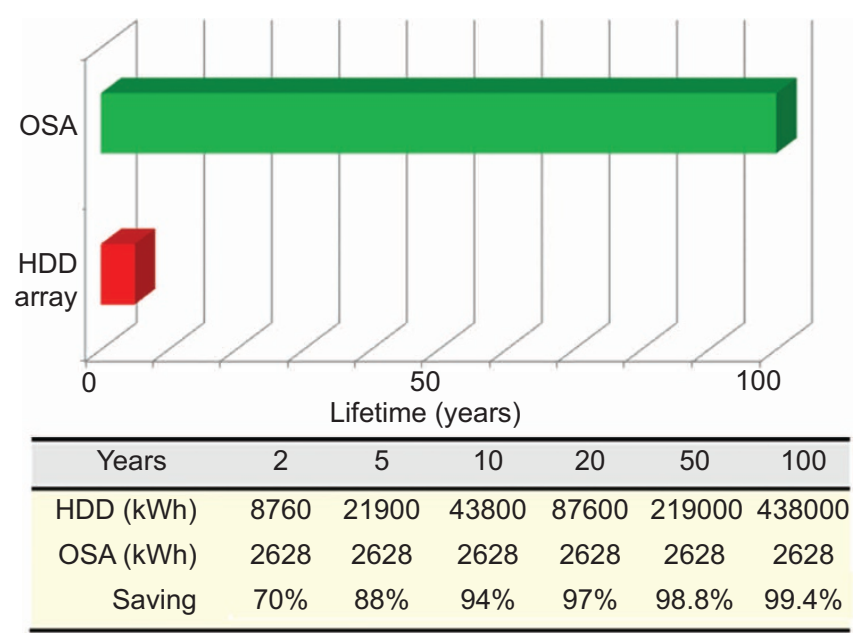

Figure 12 Comparison of typical lifetimes of OSAs (green) and HDD arrays (red) and the energy conservation offered by long-lifetime data storage and archiving. HDD, hard disk drive; OSA, optical storage array. 
and breaches of information confidence, the enriched physical dimensions of light provide exceptional protocols for the encryption information in these physical parameters instead of the conventional binary information storage offered by HDD techniques. This intriguing feature of OSAs enables orders-of-magnitude-increased flexibility in the generation of encryption keys, opening a new avenue for information storage with ultrahigh security. Moreover, OSAs allow for write-onceread-many protocols in which information, once written, cannot be modified. This type of write protection affords reliability in information storage as well as the assurance that the data cannot be tampered with once written to the storage device.

In addition, the cost for information storage can be reduced from the current price of $\sim \$ 1$ per GB to well below $\$ 0.01$ per GB by switching from HDDs to OSA techniques. Over the past two decades, ODS has proven to be a great success in the entertainment sector, with a global production of more than 2000 billion discs and a market size of multiple billions of US dollars per annum. The proposed OSA, with its exceptional capacity and energy efficiency, will become a key enabling platform for the sustainability of our digital economy and society powered by information technologies. It is reasonable to project that OSAs will gain a tremendous share in the big data technologies in the near future, with a global market on the scale of $\sim 30$ billion US dollars per annum. ${ }^{65}$ Together with the booming optical chip and optical fiber techniques, OSAs functioning as relay stations for data streams or warehouses for information archiving will be able to promote a brand-new paradigm of all-optical information management toward a sustainable future for big data storage.

\section{ACKNOWLEDGMENTS}

The authors thank the Australian Research Council for its support through the Laureate Fellowship project (FL100100099).

1 Gantz J, Reinsel D. The Digital Universe in 2020: Big Data, Bigger Digital Shadows, and Biggest Growth in the Far East. Framingham, MA: IDC; 2012. http://www. emc.com/leadership/digital-universe/index.htm.

2 Schadt EE, Linderman MD, Sorenson J, Lee L, Nolan GP. Computational solutions to large-scale data management and analysis. Nat Rev Genet 2010; 11, 647-657.

3 Trelles O, Prins P, Snir M, Jansen RC. Big data, but are we ready? Nat Rev Genet 2011; 12: 224.

4 Gu M, Li X. The road to multi-dimensional bit-by-bit optical data storage. Optics \& Photonics News 2010; 21: 28-33.

5 Parthenopoulos DA, Rentzepis PM. Three-dimensional optical storage memory. Science 1989; 245: 843-845.

6 Day D, Gu M, Smallridge A. Use of two-photon excitation for erasable-rewritable threedimensional bit optical data storage in a photorefractive polymer. Opt Lett 1999; 24: 948-950.

7 Kawata Y, Ishitobi H, Kawata S. Use of two-photon absorption in a photorefractive crystal for three-dimensional optical memory. Opt Lett 1998; 23: 756-758.

8 Day D, Gu M. Effects of refractive-index mismatch on three-dimensional optical datastorage density in a two-photon bleaching polymer. Appl Opt 1998; 37: 6299-6304.

9 Li X, Cao Y, Gu M. Superresolution-focal-volume induced 3.0 Tbytes/disk capacity by focusing a radially polarized beam. Opt Lett 2011; 36: 2510-2512.

10 Shalaev VM. Optical negative-index metamaterials. Nat Photonics 2007; 1: 41-48.

11 Chen J, Wang Y, Jia B, Geng T, Li X et al. Observation of the inverse Doppler effect in negative-index materials at optical frequencies. Nat Photonics 2011; 5: 239-245.

12 Serbin J, Gu M. Experimental evidence for superprism effects in three-dimensional polymer photonic crystals. Adv Mater 2006; 18: 221-224.

13 Chow E, Lin SY, Johnson SG, Villeneuve PR, Joannopoulos JD et al. Three-dimensional control of light in a two-dimensional photonic crystal slab. Nature 2000; 407: 983986.

14 Almeida VR, Barrios CA, Panepucci RR, Lipson M. All-optical control of light on a silicon chip. Nature 2004; 431: 1081-1084.

15 Noda S, Fujita M, Asano T. Spontaneous-emission control by photonic crystals and nanocavities. Nat Photonics 2007; 1: 449-458.

16 Li J, Jia B, Zhou G, Bullen C, Serbin J et al. Spectral redistribution in spontaneous emission from quantum-dot-infiltrated 3D woodpile photonic crystals for telecommunications. Adv Mater 2007; 19: 3276-3280.

17 Rittweger E, Han KY, Irvine SE, Eggeling C, Hell SW. STED microscopy reveals crystal colour centres with nanometric resolution. Nat Photonics 2009; 3: 144-147.
18 Rust MJ, Bates M, Zhuang X. Sub-diffraction-limit imaging by stochastic optical reconstruction microscopy (STORM). Nat Methods 2006; 3: 793-795.

19 Gu M, Cao Y, Castelletto S, Kouskousis B, Li X. Super-resolving single nitrogen vacancy centers within single nanodiamonds using a localization microscope. Opt Express 2013; 21: 17639-17646.

20 Gan Z, Cao Y, Evans RA, Gu M. Three-dimensional deep sub-diffraction optical beam lithography with 9 nm feature size. Nat Commun 2013; 4: 2061.

21 Li L, Gattass RR, Gershgoren E, Hwang H, Fourkas JT. Achieving $\lambda / 20$ resolution by one-color initiation and deactivation of polymerization. Science 2009; 324: 910 913.

22 Nikoobakht B, El-Sayed MA. Preparation and growth mechanism of gold nanorods (NRs) using seed-mediated growth method. Chem Mater 2003; 15: 1957-1962.

23 Zijlstra P, Chon JW, Gu M. Five-dimensional optical recording mediated by surface plasmons in gold nanorods. Nature 2009; 459: 410-413.

24 Li X, Chon JW, Wu S, Evans RA, Gu M. Rewritable polarization-encoded multilayer data storage in 2,5-dimethyl-4-( $p$-nitrophenylazo)anisole doped polymer. Opt Lett 2007; 32: 277-279.

25 Li X, Chon JW, Evans RA, Gu M. Quantum-rod dispersed photopolymers for multidimensional photonic applications. Opt Express 2009; 17: 2954-2961.

26 Ganic D, Day D, Gu M. Multi-level optical data storage in a photobleaching polymer using two-photon excitation under continuous wave illumination. Opt Lasers Eng 2002; 38: 433-437.

27 Zhang J. Gecevičius M, Beresna M, Kazansky PG. 5D data storage by ultrafast laser nanostructuring in glass. In: Proceedings of Conference on Lasers and Electro-Optics; 9-14 June; San Jose, CA, USA. 2013.

28 Zhang J, Gecevičius M, Beresna M, Kazansky PG. Seemingly unlimited lifetime data storage in nanostructured glass. Phys Rev Lett 2014; 112: 033901.

29 Bozinovic N, Yue Y, Ren Y, Tur M, Kristensen P et al. Terabit-scale orbital angular momentum mode division multiplexing in fibers. Science 2013; 340: 1545-1548.

30 Stanciu CD, Hansteen F, Kimel AV, Kirilyuk A, Tsukamoto A et al. All-optical magnetic recording with circularly polarized light. Phys Rev Lett 2007; 99: 047601.

31 Jiang $Y$, Li X, Gu M. Generation of sub-diffraction-limited pure longitudinal magnetization by the inverse Faraday effect through tightly focusing an azimuthally polarized vortex beam. Opt Lett 2013; 38: 2957-2960.

32 Ivanov IP, Li X, Dolan PR, Gu M. Nonlinear absorption properties of the charge states of nitrogen-vacancy centers in nanodiamonds. Opt Lett 2013; 38: 1358-1360.

33 Dolan PR, Li X, Storteboom J, Gu M. Complete determination of the orientation of NV centers with radially polarized beams. Opt Express 2014; 22: 4379-4387.

34 Castelletto S, Li X, Gu M. Frontiers in diffraction unlimited optical methods for spin manipulation, magnetic field sensing and imaging using diamond nitrogen vacancy defects. Nanophotonics 2012; 1: 139-153.

35 Balasubramanian G, Chan IY, Kolesov R, Al-Hmoud M, Tisler J et al. Nanoscale imaging magnetometry with diamond spins under ambient conditions. Nature 2008; 455: 648-651.

36 Terris BD, Mamin HJ, Rugar D, Studenmund WR, Kino GS. Near-field optical data storage using a solid immersion lens. Appl Phys Lett 1994; 65: 388-390.

37 Betzig E, Trautman JK, Wolfe R, Gyorgy EM, Finn PL et al. Near-field magneto-optics and high density data storage. App/ Phys Lett 1992; 61: 142-144.

38 Hell SW, Wichmann J. Breaking the diffraction resolution limit by stimulated emission: stimulated-emission-depletion fluorescence microscopy. Opt Lett 1994; 19: 780-782.

39 Andrew TL, Tsai HY, Menon R. Confining light to deep subwavelength dimensions to enable optical nanopatterning. Science 2009; 324: 917-921.

40 Scott TF, Kowalski BA, Sullivan AC, Bowman CN, McLeod RR. Two-color singlephoton photoinitiation and photoinhibition for subdiffraction photolithography. Science 2009; 324: 913-917.

41 Fischer J, von Freymann G, Wegener M. The materials challenge in diffractionunlimited dired-laser-writing optical lithography. Adv Mater 2010; 22: 3578-3582.

42 Cao Y, Gan Z, Jia B, Evans RA, Gu M. High-photosensitive resin for super-resolution direct-laser-writing based on photoinhibited polymerization. Opt Express 2011; 19 . 19486-19494.

43 Stocker MP, Li L, Gattass RR, Fourkas JT. Multiphoton photoresists giving nanoscale resolution that is inversely dependent on exposure time. Nat Chem 2011; 3: 223227.

44 Fischer J, Wegener M. Three-dimensional direct laser writing inspired by stimulatedemission-depletion microscopy [Invited]. Opt Mater Express 2011; 1: 614-624.

45 Harke B, Dallari W, Grancini G, Fazzi D, Brandi F et al. Polymerization inhibition by triplet state absorption for nanoscale lithography. Adv Mater 2013; 25: 904-909.

46 Wollhofen R, Katzmann J, Hrelescu C, Jacak J, Klar TA. $120 \mathrm{~nm}$ resolution and $55 \mathrm{~nm}$ structure size in STED-lithography. Opt Express 2013; 21: 10831-10840.

47 Grotjohann T, Testa I, Leutenegger M, Bock H, Urban NT et al. Diffraction-unlimited all-optical imaging and writing with a photochromic GFP. Nature 2011; 478: 204 208.

48 Cao Y, Gu M. $\lambda / 26$ silver nanodots fabricated by direct laser writing through highly sensitive two-photon photoreduction. Appl Phys Lett 2013; 103: 213104.

49 Gu M, Li X, Cao Y. Super-resolution nanolithography in photoreduction polymers. In: Proceedings of Quantum Electronics Conference \& Lasers and Electro-Optics (CLEO) IQEC/PACIFIC RIM); 28 August-1 September 2011; Sydney, NSW, Australia. IEEE: Piscataway, NJ, USA, 2011, pp1097-1098.

50 Gu M, Li X, Cao Y. Next generation photonic storage: ultra-high capacity, ultra-high security and ultra-long lifetime. In: Proceedings of IEEE Photonics Conference; 8-12 September 2013; Seattle, WA, USA. IEEE: Piscataway, NJ, USA, 2013, pp669-670. 
51 Kato JI, Takeyasu N, Adachi Y, Sun HB, Kawata S. Multiple-spot parallel processing for laser micronanofabrication. Appl Phys Lett 2005; 86: 044102.

52 Sacconi L, Froner E, Antolini R, Taghizadeh MR, Choudhury A et al. Multiphoton multifocal microscopy exploiting a diffractive optical element. Opt Lett 2003; 28 1918-1920.

53 Lin H, Jia B, Gu M. Dynamic generation of Debye diffraction-limited multifocal arrays for direct laser printing nanofabrication. Opt Lett 2011; 36: 406-408.

54 Jesacher A, Booth MJ. Parallel direct laser writing in three dimensions with spatially dependent aberration correction. Opt Express 2010; 18: 21090-21099.

55 Ren HR, Lin H, Li X, Gu M. Three-dimensional parallel recording with a Debye diffraction-limited and aberration-free volumetric multifocal array. Opt Lett 2014; 39: 1621-1624.

56 Gu M, Lin H, Li X. Parallel multiphoton microscopy with cylindrically polarized multifocal arrays. Opt Lett 2013; 38: 3627-3630.

57 Dorn R, Quabis S, Leuchs G. Sharper focus for a radially polarized light beam. Phys Rev Lett 2003; 91: 233901.

58 Wang H, Shi L, Lukyanchuk B, Sheppard C, Chong CT. Creation of a needle of longitudinally polarized light in vacuum using binary optics. Nat Photonics 2008 2: $501-505$.

59 Li X, Lan TH, Tien CH, Gu M. Three-dimensional orientation-unlimited polarization encryption by a single optically configured vectorial beam. Nat Commun 2012; 3: 998.
60 Gu M, Li X, Lan TH, Tien CH. Plasmonic keys for ultra-secure information encryption. SPIE Newsroom, 19 November 2012; doi:10.1117/2.1201211.004538.

61 Li X, Zhang Q, Chen X, Gu M. Giant refractive-index modulation by two-photon reduction of fluorescent graphene oxides for multimode optical recording. Sci Rep 2013; 3: 2819.

62 Day D, Gu M. Formation of voids in a doped polymethylmethacrylate polymer. App/ Phys Lett 2002; 80: 2404-2406.

63 Glezer EN, Milosavljevic M, Huang L, Finlay RJ, Her TH et al. Three-dimensional optical storage inside transparent materials. Opt Lett 1996; 21 : 2023-2025.

$64 \mathrm{CO}_{2}$ Berechnung, http://www.iwr.de/re/eu/co2/co2.html (accessed on 24 January 2014).

65 Vesset D, Nadkarni A, Brothers R, Christiansen CA, Conway S et al. Worldwide Big Data Technology and Services 2013-2017 Forecast. Framingham, MA: IDC; 2013.

\section{(c) (i) $(-)$ This work is licensed under a Creative Commons Attribution-}

cor NC ND NonCommercial-NoDerivs 3.0 Unported License. The images or other third party material in this article are included in the article's Creative Commons license, unless indicated otherwise in the credit line; if the material is not included under the Creative Commons license, users will need to obtain permission from the license holder to reproduce the material. To view a copy of this license, visit http://creativecommons.org/licenses/ by-nc-nd/3.0/ 